\title{
Optimal Hidden Node Area for Enhancing Routing Protocol Performance in IEEE 802.11 Multihop MANETs
}

\author{
Emeka Egbogah, Liqi Shi, and Abraham Fapojuwo \\ Department of Electrical and Computer Engineering, University of Calgary, Calgary, AB, Canada T2N 1N4 \\ Correspondence should be addressed to Emeka Egbogah, emeka.egbogah@ucalgary.ca \\ Received 5 September 2010; Accepted 9 January 2011 \\ Academic Editor: Nikos Sagias
}

Copyright (C) 2011 Emeka Egbogah et al. This is an open access article distributed under the Creative Commons Attribution License, which permits unrestricted use, distribution, and reproduction in any medium, provided the original work is properly cited.

\begin{abstract}
The prevalence of hidden node areas in IEEE 802.11 multihop MANETs continues to hinder the performance of routing protocols. This letter presents an analytical model that relates the hidden node area to the hop distance between two communicating nodes. Unlike descriptions from the literature, we describe the hidden node area in terms of multiple layers and the different levels of interference contributed by each layer. We then develop mathematical expressions to determine the probability of successful delivery and end-to-end delay of a packet transmitted over multiple hops to a receiver node exposed to hidden nodes, as a function of hop distance. The numerical results show that decreasing the hop distance increases the probability of successful packet reception at a receiver, at the cost of increased end-to-end delay. However, using a specified delay objective, routing protocols can institute a hop distance threshold metric to limit the number of transmissions that produce collisions in the hidden node area and, thus, maximize their performance.
\end{abstract}

\section{Introduction}

This paper addresses the problem of mitigating the adverse effects of hidden nodes (i.e., collisions, unfairness/starvation, rerouting instability, etc.) on the performance of routing protocols in IEEE 802.11-based multihop mobile ad-hoc networks (MANETs). We define the hidden node area as an area that is composed of multiple layers each contributing different levels of interference from interfering transmitting nodes. Using this definition, we provide an analytical model of the hidden node area that describes the relationship between the multilayered hidden node area and the hop distance between two nodes in communication. Furthermore, based on our analytical model of the hidden node area, we develop mathematical expressions for the probability of successful delivery and end-to-end delay for a packet transmitted to a receiver over multiple hops, in terms of the hop distance. The work in this letter differs from [1] because our analysis is based on a more concise definition of the hidden node area. In [1], the hidden node area is described in terms of a node's transmission range, carrier sensing range, and interference range. But in fact, the consideration of transmission range is unnecessary because it only provides the maximum allowable hop distance between nodes, which can also be expressed in terms of carrier sensing range, as is done in this letter. Moreover, [1] only considers the hidden node area as the contribution of interference from a single transmitting node. As shown in this letter, the hidden node area extends beyond a single interfering node, as it is actually composed of several interfering nodes that can be divided into different layers based on interference range. At the time of writing this letter, we discovered another paper [2] that defined the hidden node area in a similar manner to us-in terms of "interference areas" and "interference zones" with respect to the intended data recipient node. However, several assumptions are made in [2] that limit the accuracy of their work. First, the authors do not capture the exact working details of the IEEE 802.11 protocol because they assume nodes do not sense the medium before transmitting. Second, they assume all nodes within the carrier sensing range of the transmitter and inside the first interference zone of the receiver defer from transmitting, but the other nodes beyond the first zone transmit independently. These two assumptions render 
their expression for the probability of successful delivery (Equation (4) of [2]) inaccurate because it overestimates the cumulative interference at the receiver and does not account for any carrier sensing mechanism. Our analytical model accurately captures the distributed coordination function (DCF) of the 802.11 protocol by accounting for the carrier sensing mechanism of all nodes and not assuming that nodes transmit independently without considering contention. The work presented in this letter is similar to [3] in that analysis is used to show how changing the hop distance between nodes affects the network throughput in a wireless network. Specifically, [3] builds mathematical models to analyze the end-to-end throughput in networks of different scales (i.e., small: the transmission of any node can be detected by all the other nodes, large: multiple hops are required to transmit data from source to destination) as a function of hop distance and network load density. In our work, we develop a mathematical model that takes into account the effect of interfering nodes from different layers of the hidden node area on the probability of successful transmission and end-to-end delay of a packet in multihop MANETs.

\section{Hidden Node Area Model}

The relationship between the hidden node area and the distance between two nodes in communication can be derived mathematically. To do this, we first define the following two parameters.

(i) Carrier Sensing Range $\left(R_{s}\right)$ is the range within which a transmitter can detect whether or not another node is transmitting on the medium. We assume $R_{s}$ is always greater than the maximum transmission range.

(ii) Interference Range Threshold $\left(R_{i N}\right)$ is the distance at which the interference from $N$ or more interfering nodes can create packet loss at the receiving node.

The parameter $R_{i N}$ is used to describe the multiple layers that comprise the hidden node area. We can determine the expression for $R_{i N}$ by analyzing the network shown in Figure 1. In Figure 1(a), the distance between the sender $(t)$ and receiver $(r)$ is $d$, the received power of the signal from the sender at the receiver is $P_{r}$, and the interference from an interfering node located at a distance of $R_{i N}$ from the receiver is $P_{i N}$. We also assume that all nodes in the network have the same transmission power and antenna gain to simplify the analysis. Using the two ray ground reflected path loss model with thermal noise $P_{\text {noise }}$ and $N$ interfering nodes, the signal to interference plus noise ratio (SINR) at $R_{i N}$ is

$$
\operatorname{SINR}=\frac{P_{r}}{P_{\text {noise }}+N \times P_{i N}}=\frac{R_{i N}^{4}}{P_{\text {noise }}+N \times d^{4}} .
$$

Assuming the thermal noise for a $2.4 \mathrm{GHz}$ wireless channel is significantly smaller than the interference incurred from the interfering nodes, we can neglect $P_{\text {noise }}$ in (1). To correctly receive the signal, the receiver's SINR should be greater than or equal to the SINR threshold, SINR ${ }_{\text {th }}$, such that

$$
R_{i N} \geq d \times \sqrt[4]{N \times \operatorname{SINR}_{\text {th }}} .
$$

From Figure 1(a), when $N=1$ and $R_{i 1}=d \times \sqrt[4]{\operatorname{SINR}_{\text {th }}}$, the area between $R_{s}$ and $R_{i 1}$ can be defined as the first layer of the hidden node area, within which a single interfering node in transmission mode can cause packet loss at the receiver. To generalize, the area between $R_{i N-1}$ and $R_{i N}(N>1)$ can be defined as the $N$ th layer of the hidden node area, within which $N$ or more nodes in transmission mode can cause packet loss at the receiver. However, at a certain distance $R_{i M}$ (where $M$ is the last layer of the hidden node area) the interference from a single node is so small that it can be neglected at the receiver (i.e., $P_{R_{i M}} \ll P_{i}$ ). For that reason, the size of the hidden node area is upper bounded by a distance $R_{i M}$, where the interference power is negligible at the receiver. At $R_{i M}$, the number of nodes in transmission mode should be less than $M$ and separated by a distance of at least $R_{s}$ (to ensure they are outside of each other's sensing range) to avoid packet loss at the receiver. Therefore, as $M$ increases, the probability of $M$ interfering nodes simultaneously transmitting and creating packet loss at the receiver becomes smaller. Thus, the transmitting node(s) in the first layer of the hidden node area can be deemed as having the most significant hidden node effect.

The maximum transmission range of a node using the standard IEEE 802.11 MAC protocol is less than the carrier sensing range [4]. Also, the node-to-node communication distance $d$ (also referred to as hop distance) should be less than the upper-bound size of the hidden node area $\left(R_{i M}\right)$ to avoid significant packet loss at the receiver. Therefore, it is reasonable to assume that $d$ is less than both $R_{s}$ and $R_{i M}$. Using this assumption, the hidden node area $\left(A_{h}\right)$ is calculated in terms of the hop distance $d$ under three different scenarios where the value of $R_{s}$ (or $d$ ) exists in different ranges due to the mobility of nodes in MANETs.

Scenario 1 (for $R_{s}$ depicted in Figure 1(a)). The carrier sensing range is adaptive and can lie below or above the hidden node area threshold distance such that $\left(R_{i M}-\right.$ $d) \leq R_{s} \leq\left(R_{i M}+d\right)$. Or, in terms of hop distance, $R_{s} /\left(\sqrt[4]{M \times \text { SINR }_{\mathrm{th}}}+1\right) \leq d \leq R_{s} /\left(\sqrt[4]{M \times \text { SINR }_{\mathrm{th}}}-1\right)$. The hidden node area can then be calculated as

$$
A_{h}=\pi R_{i M}^{2}-\alpha R_{s}^{2}-\beta R_{i M}^{2}+R_{i M} d \sin \beta,
$$

where $\beta=\cos ^{-1}\left(d^{2}+R_{i M}^{2}-R_{s}^{2}\right) /\left(2 \times d \times R_{i M}\right)$, and $\propto=$ $\cos ^{-1}\left(d^{2}+R_{s}^{2}-R_{i M}^{2}\right) /\left(2 \times d \times R_{s}\right)$.

Applying (2) to (3) gives

$$
A_{h}=\left(\pi-\beta+\frac{\sin \beta}{\sqrt[4]{M \times \operatorname{SINR}_{\mathrm{th}}}}\right) \sqrt{M \times \operatorname{SINR}_{\mathrm{th}}} \times d^{2}-\alpha R_{s}^{2} .
$$

Scenario 2 (for $R_{s}$ depicted in Figure 1(b)). The carrier sensing range is always larger than the hidden node area threshold distance such that $R_{s}>\left(R_{i M}+d\right)$, or $d<$ $R_{s} /\left(\sqrt[4]{\operatorname{SINR}_{\text {th }} \times M}+1\right)$. Obviously, $A_{h}$ is zero under this scenario.

Scenario 3 (for $R_{s}$ depicted in Figure 1(c)). The carrier sensing range is always smaller than the hidden node area 
Hidden node area

First layer $(N=1)$

Second layer $(N=2)$

28 Third layer $(N=3)$

$\because$ Remaining area $\left(P_{\text {Rhn }} \ll P_{r}\right)$

Carrier sensing range of transmitter

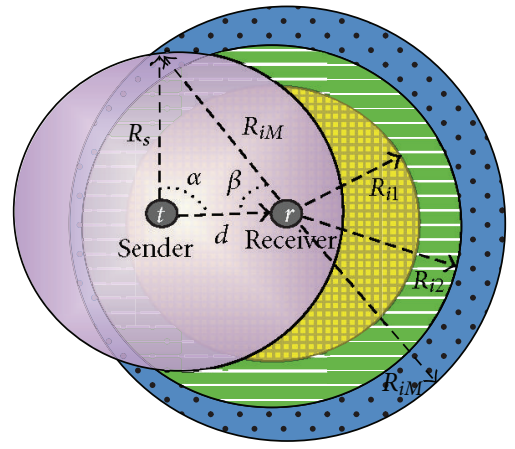

(a)

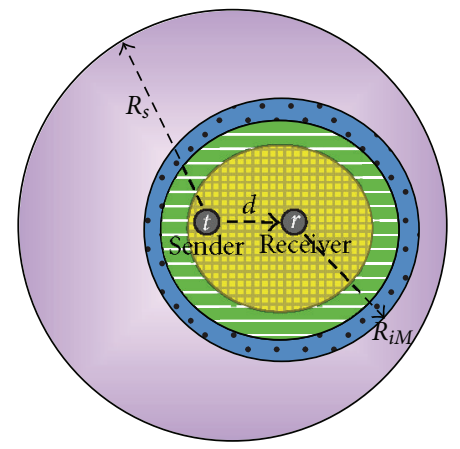

(b)

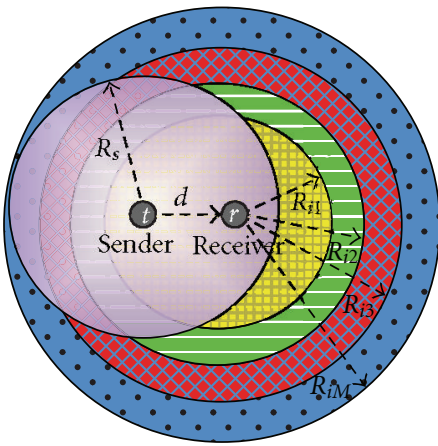

(c)

Figure 1: Hidden node area under different conditions.

threshold distance such that $R_{s}<\left(R_{i M}-d\right)$, or $d>$ $R_{s} /\left(\sqrt[4]{\operatorname{SINR}_{\mathrm{th}} \times M}-1\right)$. The hidden node area is then

$$
A_{h}=\pi\left(\sqrt{M \times \operatorname{SINR}_{\mathrm{th}}} \times d^{2}-R_{s}^{2}\right) .
$$

From (4) and (5), it is shown that the hidden node area increases as the hop distance squared, under the assumption of the two-ray ground reflected path loss model. Conversely, as the hop distance between the sender and receiver decreases, the average received signal strength increases. So, for a single hop, the probability of successful delivery $P_{s}$ is improved as the hop distance decreases. However, reducing the hop distance also adversely affects the endto-end communication performance along a multihop path because it increases the average number of hops along a path and reduces the number of available next hop candidates at every intermediate node. Increasing the average number of hops along a path leads to increased end-toend transmission delay, and a reduction in the number of available intermediate nodes has the potential to make data packet delivery less robust because there are fewer delivery paths.

Based on the preceding observation, we claim that endto-end communication in IEEE 802.11 multihop MANETs achieves the best tradeoff between $P_{s}$ and end-to-end transmission delay at a certain hop distance where the delay objective is satisfied. To prove this claim, we develop an analytical model that describes the relationship between $P_{s}$, delay, and hop distance in relation to the hidden node area in the next section.

\section{Mathematical Expressions for $P_{s}$ and End-to-End Delay}

3.1. Probability of Successful Delivery, $P_{s}$. The probability of successful delivery is determined by estimating the probability that a collision does not occur when a data packet is transmitted from a sender to an intended receiver. We assume all collisions are a result of interference from transmitting nodes (hereafter referred to as interferers) located in different layers of the hidden node area.

In this paper, we use a probabilistic approach to randomly identify the existence of interferers. In practice, virtual carrier sensing techniques such as the exchange of Request-To-Send (RTS) and Clear-To-Send (CTS) messages in IEEE 802.11 can be used to prevent hidden nodes from transmitting and consequently causing collisions and interference [5]. However, the use of RTS/CTS results in the proliferation of the exposed node problem, where certain nodes that had heard the exchange of RTS/CTS messages refrain from transmitting even though they would not have interfered with any other ongoing transmissions. Accounting for the RTS/CTS mechanism and its effect on the hidden node problem is beyond the scope of this paper. However, a further detailed explanation of the RTS/CTS mechanism and its effect on the hidden problem is provided in [5].

To estimate the probability that a collision does not occur when a sender transmits a packet to the receiver while $i_{N}$ interfering nodes exist at each layer $N$ of the hidden node area, all combinations for the selection of the number of eligible interfering nodes must be considered, where $i_{N} \leq(N-1)$ (e.g., for $M=3,\left(i_{1}, i_{2}, i_{3}\right)=$ $\{(0,0,0),(0,0,1),(0,0,2),(0,1,0),(0,1,1),(0,1,2)\})$. The interferer(s) are randomly selected from each layer and 
assigned the same transmission probability of $\gamma$. However, the following two constraints must be placed on each combination of interferes to accurately account for the carrier sense multiple access (CSMA) mechanism of the IEEE 802.11 protocol.

(C1) For each combination of selected interferers, each interferer must be outside of the sensing range of the other selected interferers.

(C2) The total received interference power $\left(\sum_{N=1}^{M} P_{i N}\right)$ of the signals from the selected interferers must be less than a certain threshold $P_{i_{\mathrm{th}}}\left(P_{i_{\mathrm{th}}}=\operatorname{SINR}_{\mathrm{th}} \times N \times P_{i}\right)$ so that interference does not cause packet loss at the receiver.

Using the two ray ground reflected path loss model, the received power from an interferer at layer $N$ can be modeled as [6]

$$
P_{i N}=P_{t} G_{t} G_{r} \frac{h_{t}^{2} h_{r}^{2}}{d_{i N}^{4}},
$$

where $P_{t}$ is the transmission power, $G_{t}$ and $G_{r}$ are the antenna gains of the transmitter and receiver, respectively, $h_{t}$ and $h_{r}$ are, respectively, the heights of both transmit and receive antennas, and $d_{i N}$ is the distance between an interferer and the receiver.

Assume the transmitting probability at a node is $\gamma$. The probability that $i_{N}$ nodes are active in the $N$ th layer is $\gamma^{i_{N}}(1-\gamma)^{r_{N}}$, where $r_{N}$ is the remaining nodes in layer $N$, given by

$$
r_{N}=\rho X\left(A_{l, N} \cap A_{h, N}\right) .
$$

In (7), $\rho$ is the network node density, $A_{l, N}$ is the area of the $N$ th hidden node layer, $A_{h, N}$ is the entire hidden node area between layers 1 and $N$ excluding the area outside the sensing range of the interfering nodes. Using the constraints and definitions provided in this section, the probability of successful delivery, $P_{s}$, when a sender transmits a packet to a receiver node, can be expressed as

$$
\begin{aligned}
P_{s}= & \sum_{\forall\left\{i_{j}\right\}_{j=1}^{M}} \gamma^{i_{1}}(1-\gamma)^{r_{1}} \gamma^{i_{2}}(1-\gamma)^{r_{2}} \\
& \quad \times \gamma^{i_{3}}(1-\gamma)^{r_{3}} \cdots \gamma^{i_{M}}(1-\gamma)^{r_{M}},
\end{aligned}
$$

where for all $\left\{i_{j}\right\}_{j=1}^{M}$ denotes all possible combinations of $i_{1}, i_{2}, \ldots, i_{M}$.

The probability of successful delivery is directly related to the size of the hidden node area, which is directly related to the hop distance $d$. For a multihop MANET, the probability of successful delivery of a packet transmitted from a source to destination node separated by $H$ hops, given $X$ retransmissions, is

$$
\begin{gathered}
P_{s, \text { multihop }}=\left(\left(1-P_{s}\right)^{X} P_{s}\right)^{H}, \\
X=0,1,2, \ldots 6, \quad H=1,2,3, \ldots .
\end{gathered}
$$

TABLE 1: Selected input parameter values [4].

\begin{tabular}{lc}
\hline Parameter & Value \\
\hline Packet payload $($ PLD) & $4096 \mathrm{bits}$ \\
UDP header & 64 bits \\
IP header & 160 bits \\
LLC + MAC header & 192 bits \\
PHY header & 192 bits \\
CW $_{\text {min }}$ & 32 \\
Channel bit rate & $2 \mathrm{Mbps}$ \\
Propagation delay $\left(t_{\text {prop }}\right)$ & $1 \mu \mathrm{s}$ \\
DIFS & $50 \mu \mathrm{s}$ \\
$T_{\text {slot }}$ & $20 \mu \mathrm{s}$ \\
\hline
\end{tabular}

In Figure 2, an illustrative example is provided to show how to calculate $i_{N}, r_{N}$, and $P_{s}$ for (8). In Figure 2(a), the hidden node area of the receiver $(r)$ is comprised of 3 layers $(M=3)$, in which layer 1 has 3 nodes and $i_{1}=0$, layer 2 has 4 nodes and $i_{2}=1$ (node 4 ), and layer 3 has 5 nodes and $i_{3}=2$ (nodes 10 and 12). In Figure 2(b), $A_{h, 3}$ is the entire hidden node area comprised of layers 1 to 3 , excluding the area outside the sensing range of the transmitting interfering nodes (nodes 4, 10, and 12). In Figure 2(c), the resulting area of $A_{l, 3} \cap A_{h, 3}$ is shown. In this area, $\rho X\left(A_{l, 3} \cap A_{h, 3}\right)$ results in $r_{3}=2$ (nodes 8 and 9 ). Node 11 is excluded from the calculation for $r_{3}$ because it falls within the sensing range of the interfering node (node 10). Using (8) for the specific combinations shown in Figure $2(\mathrm{a})$, where $i_{1}=0, i_{2}=1$, and $i_{3}=2$, the probability of successful delivery at the receiver, for this combination, can be calculated as $P_{s}\left(i_{1}=0, i_{2}=1, i_{3}=2\right)=$ $\gamma^{0}(1-\gamma)^{3} \gamma^{1}(1-\gamma)^{2} \gamma^{2}(1-\gamma)^{2}$.

3.2. Average End-to-End Delay. We determine the average end-to-end delay as a function of hop distance. Our analysis considers the basic access packet transmission scheme employed by the DCF of IEEE 802.11 with binary slotted exponential backoff. Table 1 lists the parameter values used for the basic DCF access mode [4]. Let HDR = PHY + MAC + IP + UDP be the packet header, PLD the packet payload, and $t_{\text {prop }}$ the propagation delay for a packet. In the basic access mode, the time to transmit a packet at its first transmission attempt is [4]

$$
T S_{0}=\frac{\mathrm{HDR}+\mathrm{PLD}}{\text { channel bit rate }}+\mathrm{DIFS}+t_{\text {prop }}
$$

By default, DCF is configured for retransmissions [4]. DCF adopts an exponential backoff scheme in which the contention window $w$ is doubled after each unsuccessful transmission. At the first transmission attempt, $w$ is set equal to a value $\mathrm{CW}_{\text {min }}$ called the minimum contention window. The average exponential backoff time after the $n$th transmission attempt, $B_{n}$, can then be expressed as

$$
B_{n}=T_{\text {slot }} \times \mathrm{CW}_{\min } \times \frac{2^{n-1}}{2} .
$$

Combining (9) and (10), $T S_{n}=T S_{n-1}+B_{n}(n>1)$. When estimating the delay, we do not consider all the mechanisms 


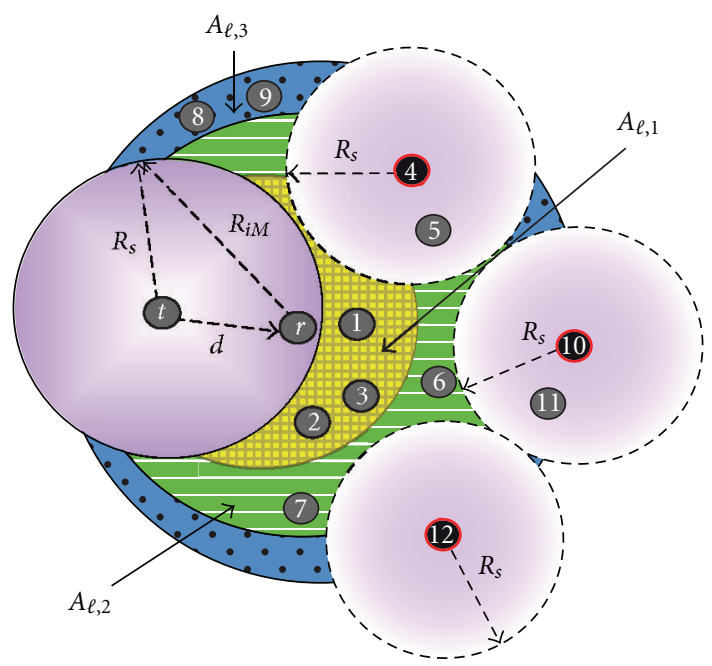

(a)

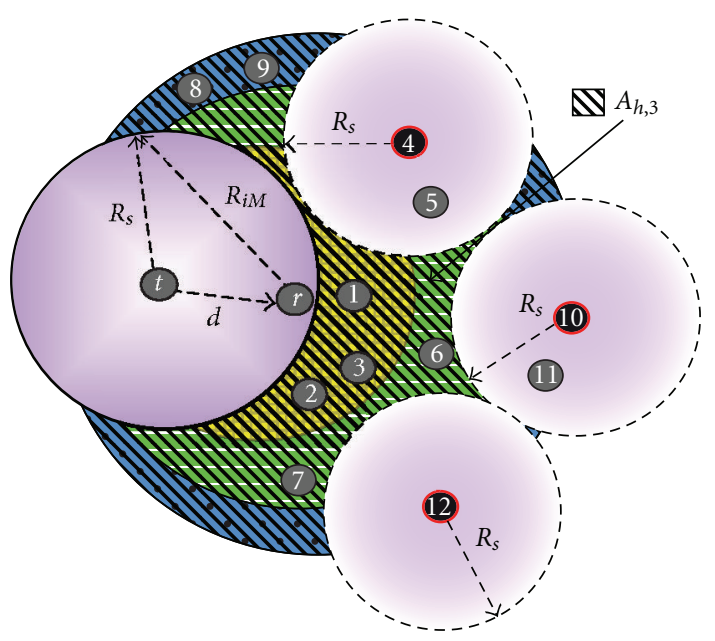

(b)

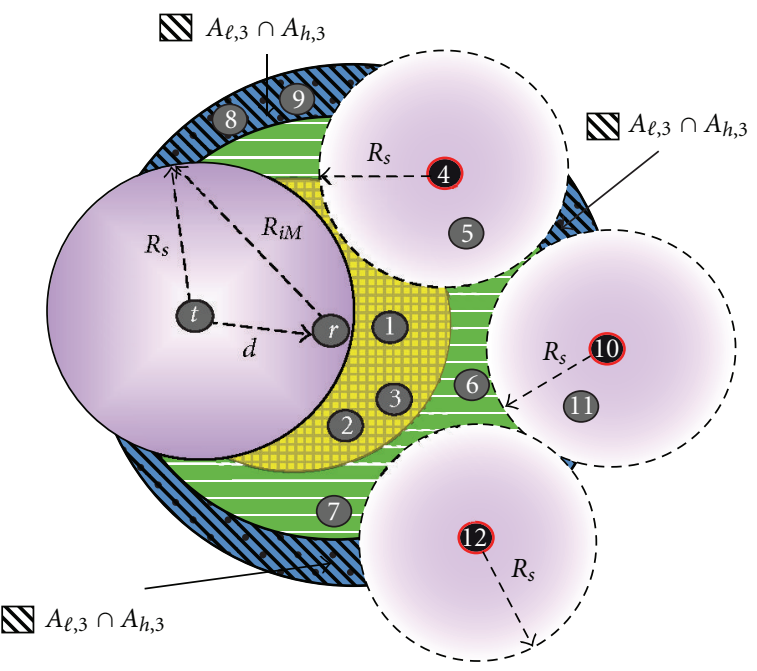

(c)

FIgURE 2: Illustrative example for calculating $i_{N}, r_{N}$, and $P_{s}$.

for contending the medium for two main reasons. (1) The proportion of the total transmission duration required of measuring the delay due to collisions is far greater than the time required to access the medium because collisions are dominant. (2) We use a small transmission probability similar to [2], otherwise the rate of collision becomes too high, as shown in the numerical results in Section 4. This assumption allows any packet queued at the sender to be immediately sent to the receiver. Using the expression for probability of successful delivery from (8) and modeling our delay expression with retransmissions, the average end-toend delay can be calculated as

$$
T_{d}=H \times\left(\sum_{n=0}^{X}\left(1-P_{s}\right)^{n} \times P_{s} \times T S_{n}\right) .
$$

In (12), the average end-to-end delay is expressed as a function of $P_{s}$. From (8), $P_{s}$ is directly related to the size of the hidden node area, which is directly related to the hop distance $d$. Therefore, $T_{d}$ is a function of hop distance $d$ via $P_{s}$.

\section{Numerical Results and Discussion}

This section presents numerical results for the probability of successful delivery and average end-to-end delay for a data packet transmitted over multiple hops to a receiver as a function of hop distance, under conditions where the hidden nodes are prevalent. MATLAB [6] is used to analyze the mathematical expressions provided in (8) and (12). We have simulated a MANET consisting of 100 nodes, randomly distributed in a $1500 \mathrm{~m} \times 1500 \mathrm{~m}$ area. 1000 random network topologies (i.e., network scenarios) were simulated, where a network topology is defined by each instance of the 100 locations of the mobile nodes. For each network scenario, the carrier sensing range is set constant to $670 \mathrm{~m}$, as specified in the IEEE 802.11 standard [4]. We 


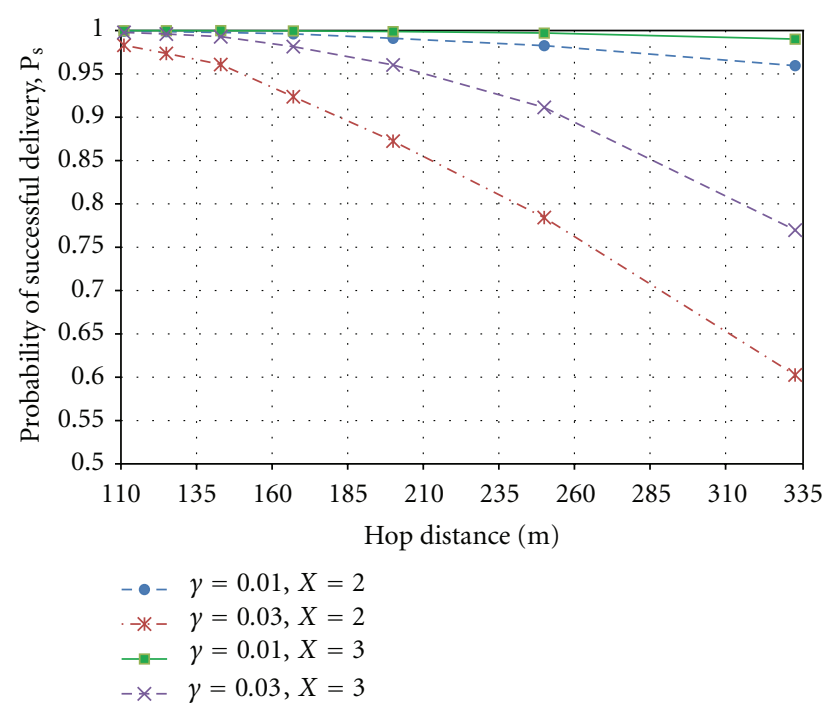

FIgURe 3: Probability of successful delivery versus hop distance.

configure DCF to handle up to 4 transmissions $(X=3)$ because we use long data packets [4].

For transmission probabilities $(\gamma)$ of 0.01 and 0.03 , and retransmissions $(X)$ of 2 and 3 for each transmission probability, Figure 3 shows the analytical results for the probability of successful delivery as the number of hops and hop distance are varied over a source-destination distance of $1000 \mathrm{~m}$. It is observed from Figure 3 that, as the hop distance increases, the probability of successful delivery decreases. The explanation is as follows. With an increase in the hop distance, the hidden node area becomes larger and consequently, more active interfering nodes exist, causing collisions at the receiver, resulting in lower probability of successful reception. As the hop distance decreases, the probability of successful delivery to a receiver increases. However, when the hop distance is less than a certain distance, the benefits earned from a small hop distance are reduced. For example, in Figure 3, when the hop distance is between $111 \mathrm{~m}$ and $167 \mathrm{~m}$ for $\gamma=0.03$ and $X=2$, the $P_{s}$ only differs by approximately $6 \%$. From (5), it is seen that the hidden node area $A_{h}$ is proportional to the hop distance squared $\left(d^{2}\right)$. At lower values of hop distance $(d<167 \mathrm{~m})$, fewer interferers exist and the size of the hidden node area does not change much, thus resulting in a more stable $P_{s}$. On the other hand, at higher values of $d$ the hidden node area increases dramatically. There are large numbers of interferers at higher values of $d$ than at lower values, resulting in a sharp drop of $P_{s}$ at the higher values of $d$, as shown in Figure 3 for the hop distances between $200 \mathrm{~m}$ and $333 \mathrm{~m}$.

Figure 3 shows that even though a hop distance of $143 \mathrm{~m}$ (7 hops $=1000 \mathrm{~m} /(143 \mathrm{~m} / \mathrm{hop})$ ) requires 4 more hops to reach the destination than a hop distance of $333 \mathrm{~m}$ ( 3 hops $=$ $1000 \mathrm{~m} /(333 \mathrm{~m} / \mathrm{hop}))$, the probability of successful delivery is approximately $35 \%$ more when $\gamma=0.03$ and $X=2$. Another trend that can be observed from Figure 3 is that the probability of successful delivery decreases as the transmission probability increases. This trend is expected because,

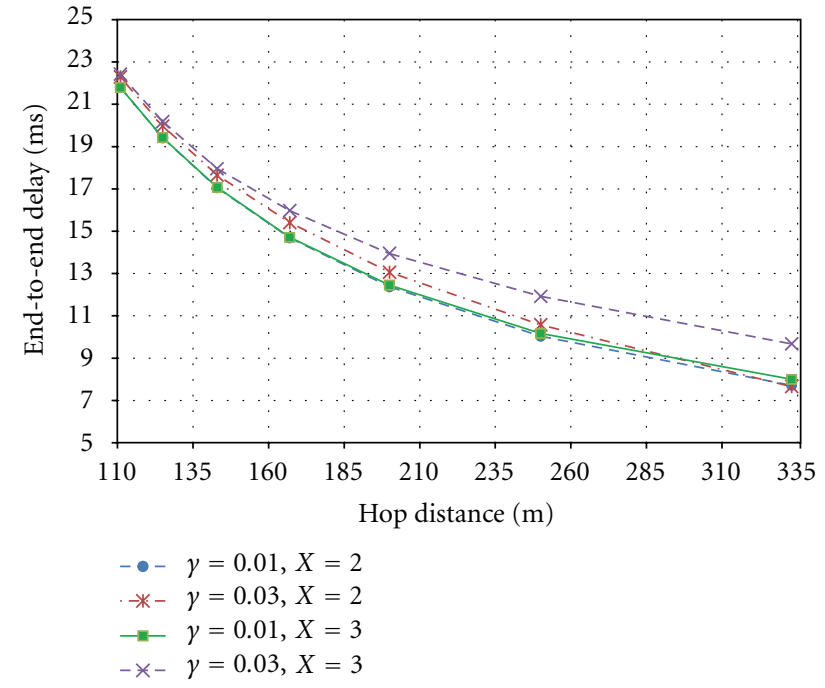

Figure 4: Average end-to-end delay versus hop distance.

as the transmission probability increases, more packets are transmitted in the network and thus the probability of collisions due to the existence of hidden nodes increases. The results in Figure 3 also show that, at a given hop distance, increasing the number of retransmissions from 2 to 3 improves the probability of successful delivery. The unpredictable nature of the wireless medium in MANETs requires that the DCF of the IEEE 802.11 protocol use retransmissions to improve the probability of delivery, and thus, we show the precise benefits of different values of $X$. Equation (9) shows that as the number of retransmissions increases, the overall probability of delivery also increases, as shown in Figure 3.

The analytical results for the average end-to-end delay over multiple hops are shown in Figure 4. As the hop distance increases, the number of hops decreases which results in a decrease of end-to-end delay (from (12)). As expected, with an increase in transmission probability and retransmissions, the end-to-end delay also increases. Figures 3 and 4 demonstrate the tradeoff existing between probability of successfully delivery and end-to-end delay as the hop distance (or number of hops to the destination) is varied: at a low hop distance and larger hop count to the destination, the probability of successful delivery is higher due to a smaller hidden node area (Figure 3), but this is at the expense of increased end-to-end delay due to the high number of hops $H$ (Figure 4). The two figures are, therefore, useful for determining the optimum hop distance for IEEE 802.11 multihop MANETs at a specified delay objective.

\section{Conclusion}

We have defined a multi-layer hidden node area model by accounting for the collective interference caused by the transmissions of multiple nodes, each located at a different distance. Using this model, the relationship between the hop distance between communicating nodes and the hidden 
node area is mathematically expressed in terms of the carrier sensing range and interference range. Through analysis, it is determined that decreasing the hop distance between a source and destination node separated by multiple hops increases the probability of successful packet delivery to a receiver. However, reducing the hop distance also increases the number of hops and thus increases the average endto-end delay. It is concluded that a tradeoff exists between the probability of successful delivery and average end-toend delay. For certain quality-of-service- (QoS-) oriented applications where a delay objective must be met, the numerical results from this paper can be used as a basis to develop a hop distance threshold (HDT) metric for routing protocols to limit the number of transmissions that occur in the hidden node area. The work presented in this paper can be extended by implementing the HDT metric in routing protocols that exist in the literature and to assess the benefit of using the HDT as a routing metric.

\section{References}

[1] K. Xu, M. Gerla, and S. Bae, "How effective is the IEEE 802.11 RTS/CTS handshake in ad hoc networks?" in Proceedings of the IEEE Global Telecommunications Conference (GLOBECOM '02), vol. 1, pp. 17-21, November 2002.

[2] G. Parissidis, M. Karaliopoulos, M. May, T. Spyropoulos, and B. Plattner, "Interference in wireless multihop networks: a model and its experimental evaluation," in Proceedings of the IEEE International Symposium on a World of Wireless, Mobile and Multimedia Networks (WoWMoM '08), Newport Beach, Calif, USA, June 2008.

[3] X. Wu, G. Ding, and W. Zhu, "Load-based route discovery through searching range adaptation for MANET throughput improvement," IEEE Transactions on Vehicular Technology, vol. 58, no. 4, pp. 2055-2066, 2009.

[4] IEEE Standards Board, "Wireless LAN Medium Access Control (MAC) and Physical Layer (PHY) Specifications," The Institute of Electrical and Electronics Engineers, Inc., November 1997.

[5] A. Jayasuriya, S. Perreau, A. Dadej, and S. Gordon, "Hidden vs. exposed terminal problem in ad hoc networks," in Proceedings of the Australian Telecommunication Networks and Applications Conference, Sydney, Australia, 2004.

[6] T. S. Rappaport, Wireless Communications, Principles and Practice, Prentice-Hall, New York, NY, USA, 2nd edition, 2004. 

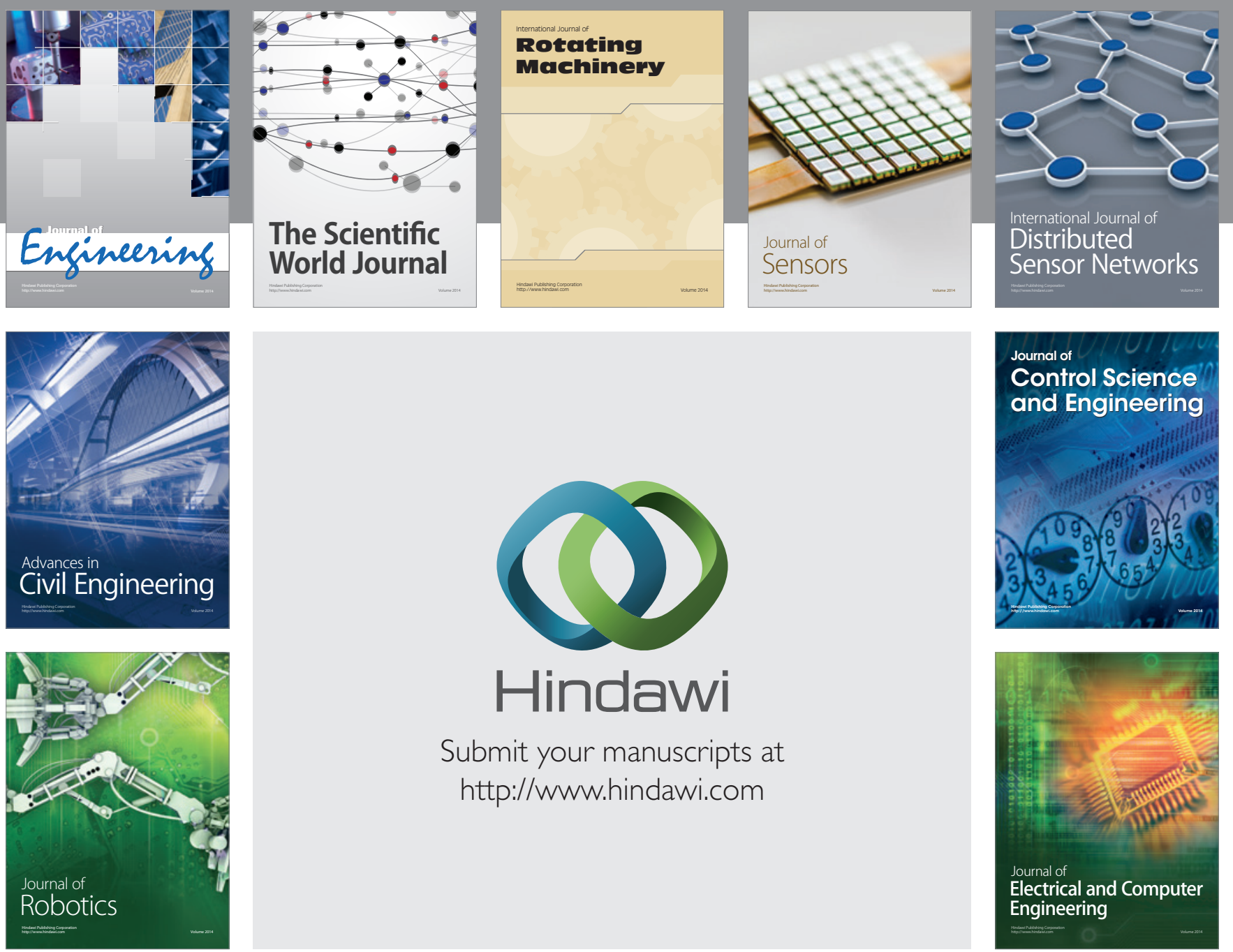

Submit your manuscripts at

http://www.hindawi.com
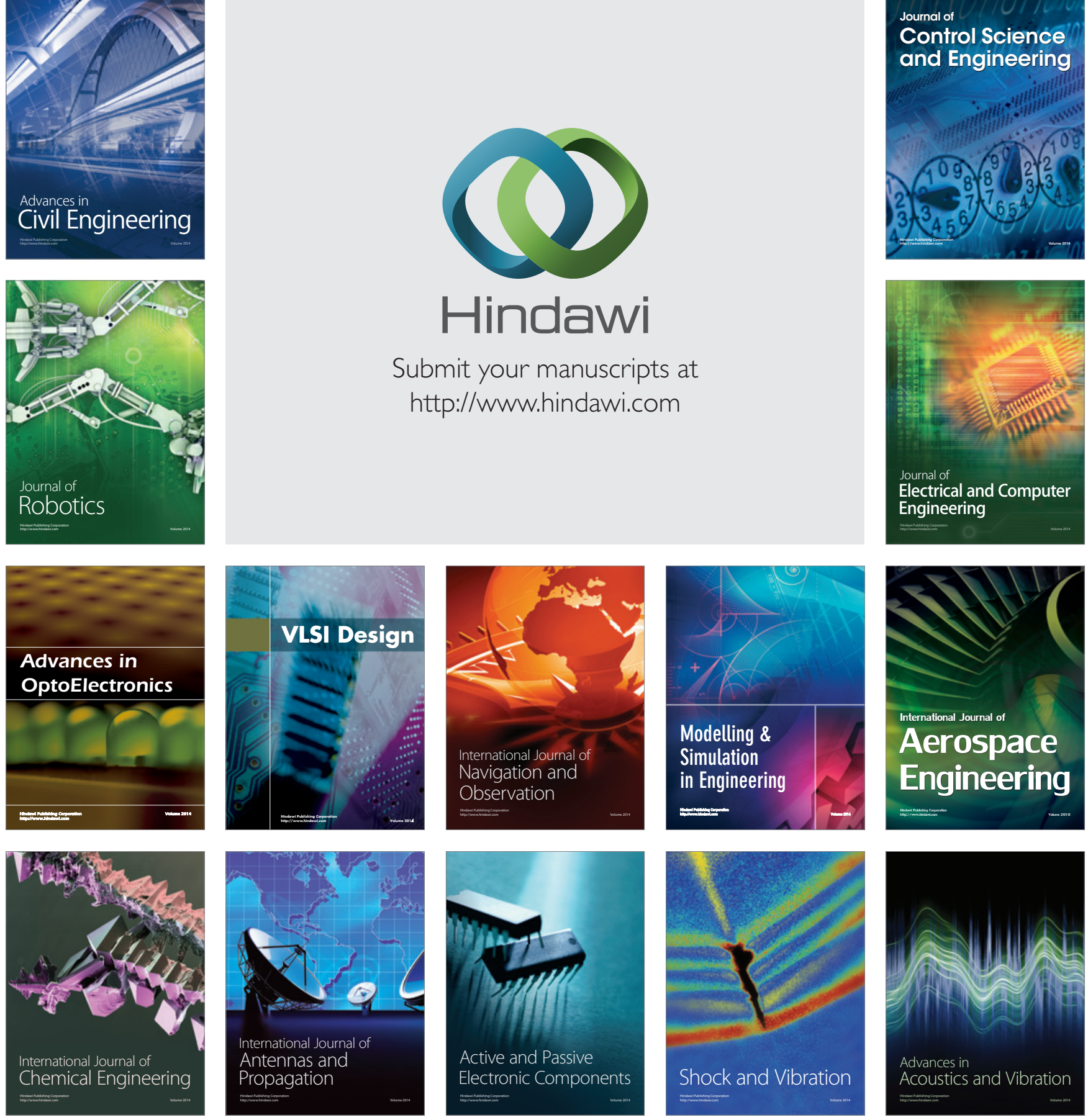